\title{
THE APRIL MEETING IN OAK RIDGE
}

The four hundred fifty-sixth meeting of the American Mathematical Society was held at Oak Ridge, Tennessee on Friday and Saturday, April 21-22, 1950, in conjunction with a meeting of the Biometric Society. The total attendance at the meeting was one hundred fifty-three, including the following eighty-seven members of the American Mathematical Society:

G. E. Albert, R. E. Allan, Herman Betz, R. H. Bing, M. G. Boyce, A. T. Brauer, R. C. Bullock, P. B. Burcham, Robert Butz, C. L. Carroll, C. E. Clark, J. M. Clarkson, A. C. Cohen, J. A. Cooley, R. R. Coveyou, J. C. Currie, L. J. Derr, N. M. Dismuke, W. L. Duren, Patrick DuVal, E. L. Eagle, E. D. Eaves, M. E. Estill, G. M. Ewing, F. A. Ficken, H. A. Fisher, Tomlinson Fort, G. A. Garrett, J. R. Garrett, Wallace Givens, A. W. Goodman, E. H. Hadlock, J. C. Harden, O. G. Harrold, I. R. Hershner, A. K. Hinds, A. S. Householder, S. T. Hu, G. B. Huff, Ralph Hull, R. B. Johnson, A. M. Jones, V. L. Klee, F. W. Kokomoor, G. B. Lang, C. G. Latimer, H. L. Lee, R. J. Levit, F. A. Lewis, Z. L. Loflin, Nathaniel Macon, W. R. Mann, J. M. Marr, E. P. Miles, D. D. Miller, Lewis Nelson, O. M. Nikodým, W. V. Parker, C. R. Partington, C. G. Phipps, E. E. Posey, Anatol Rapoport, L. T. Ratner, E. K. Ritter, M. Ethyl Rivers, J. H. Roberts, L. V. Robinson, Walter Rudin, W. A. Rutledge, J. W. Sawyer, E. B. Shanks, Seymour Sherman, Annette Sinclair, M. B. Sledd, C. B. Smith, W. S. Snyder, C. T. Taam, W. B. Temple, J. M. Thomas, W. R. Utz, A. D. Wallace, J. A. Ward, W. M. Whyburn, Ernest Williams, R. L. Wilson, E. A. Winter.

At 8:00 P.M. on Friday evening, by invitation of the Committee to Select Hour Speakers for Southeastern Sectional Meetings, addresses were delivered by Professor Alfred Brauer, University of North Carolina, and Professor W. V. Parker, Alabama Polytechnic Institute. The title of Professor Brauer's address was Criteria for the irreducibility of algebraic equations, and that of Professor Parker, Characteristic roots and field of values of a matrix. Professor Wallace Givens presided during these addresses.

Four sessions were held for contributed papers: Friday, 10:30 A.M., Dr. A. S. Householder presiding; Friday, 2:00 P.M., Professor O. G. Harrold presiding; Saturday, 9:30 A.M., Professor Ralph Hull presiding; and Saturday, 11:15 A.M., Professor C. G. Latimer presiding.

The Biometric Society held a session at 4:00 P.M. on Friday at which the following addresses were presented: The thermodynamics and steady-state kinetics of some biological systems by Professor J. Z. Hearon, Probabilistic theory of neural nets by Professor Anatol Rapoport, Mathematical basis of the interpretation of isotope experiments by Dr. C. W. Sheppard. 
Abstracts of papers presented at the meeting are given below. Papers read by title are indicated by the letter " $t$." Paper number 278 was read by Mr. Hinds, and paper number 284 was read by Professor Roberts. Mr. Hopper was introduced by Professor Wallace Givens, Mr. Butz by Professor Tomlinson Fort, Messrs. Conner and Keesee by Mr. R. E. Allan, and Mr. Derr by Professor A. D. Wallace.

\section{Algebra AND Theory of Numbers}

\section{R. K. Butz: On 2-commutative matrices.}

The methods used by W. V. Parker for writing the matrix $X$ such that $A X=X B$ (Bull. Amer. Math. Soc. Abstract 55-7-287) are extended to solve the problem of finding a matrix $X$ with respect to which a given matrix $A$, with elements in any field $F$, is 2-commutative. When $A$ is in rational canonical form and $X$ is partitioned properly, these methods display $X$ explicitly in terms of parameters. (Received March 9, 1950.)

\section{1t. Leonard Carlitz: $A$ theorem on higher congruences.}

This note contains a proof of the theorem that if the congruence $f(x) \equiv 0\left(\bmod p^{r}\right)$ is solvable for $r=\delta+1$, where $p^{\delta}$ is the highest power of $p$ dividing the discriminant of $f(x)$, then the congruence is solvable for all $r$. While the theorem is contained in a more general theorem of Hensel a direct proof seems of interest. (Received March 6, 1950.)

\section{2t. Leonard Carlitz: Some special functions connected with} $G F\left(p^{n}, x\right)$.

This paper is concerned with the properties of the function $g(u, t)$ $=\sum_{0}^{\infty} \psi_{i}(u) \phi_{i}(t) / F_{i}$ defined in Duke Math. J. vol. 15 (1948) p. 1008, and some related functions. (Received March 6,1950.)

\section{3t. Leonard Carlitz: Sums of kth powers in $G F\left[p^{n}, x\right]$.}

In this paper an asymptotic formula is obtained for the number of solutions of $M=\alpha_{1} X_{1}^{k}+\cdots+\alpha_{s} X_{s}^{k}$, where $\alpha_{j} \in G F\left(p^{n}\right) ; M, X_{j} \in G F\left[p^{n}, x\right]$, deg $M \leqq m k$, deg $X_{j}=m, X_{j}$ primary, $j \leqq l ; \operatorname{deg} X_{i}<m, i>l$, and $1 \leqq l \leqq s ;$ finally $s \geqq s_{0}(k)$. The method is that developed in Duke Math. J. vol. 14 (1947) pp. 1121-1137; vol. 15 (1948) pp. 795-801. The case $l=0$ is excluded. (Received March 6, 1950.)

\section{J. C. Currie: Unitary-canonical matrices. Preliminary report.}

It is known (I. Schur, Über die charakteristischen Wurzeln einer linearen Substitution, Math. Ann. vol. 66 (1909) pp. 488-510) that for any matrix $A$ there exists a unitary matrix $U$ such that $U A \bar{U}^{\prime}$ is triangular. However, the matrix $U$ which performs this transformation is not unique, and the resulting triangular form is not unique. In this note it is established that from the set of matrices which are unitary transforms of a given matrix $A$ a particular triangular matrix can be designated as the canonical representative of the set. Let the characteristic roots of $A$ be ordered so that if $m_{j}$ is the nullity of $\lambda_{j} I-A$, then $m_{j} \geqq m_{j+1}, j=1, \cdots, n-1$. Then $A$ is unitarily transformed into a triangular matrix in which square submatrices of the form $\lambda_{j} I_{j}$ (where $I_{j}$ 
is of order $m_{j}$ ) appear in the principal diagonal. Additional zeros are introduced above the principal diagonal in an orderly procedure such that the end result is a triangular matrix with a maximum number of zeros above the principal diagonal, the zeros being systematically placed so that the form is unique for a given $A$. (Received March 6, 1950.)

265. J. R. Garrett: Normal equations and resolvents in fields of characteristic $p$.

The general equation of degree not less than 5 with coefficients in a field $F$ of characteristic $p$ is reduced to the so-called principal and normal forms by means of Tschirnhaus transformations. In addition, a sextic resolvent is obtained for the normal quintic when $p=2,3$, and 5 . For corresponding results when $F$ is of characteristic zero see Dickson's Modern algebraic theories, chap. 12. (Received March 6, 1950.)

\section{Wallace Givens: $A$ Hadamard bound theorem for the de- terminant of a real guaternion matrix.}

The determinant of a regular matrix $A$ of order $n$ with elements in a division ring $K$ is defined by Dieudonné [Bull. Soc. Math. France vol. 71 (1943) pp. 27-45] to be an element $\Delta(A)$ of the quotient group of the multiplicative group of nonzero elements of $K$ with respect to its commutator subgroup. If one specializes to real quaternions, the group of determinantal values is isomorphic to the non-negative real numbers (with $\Delta(A)=0$ if and only if $A$ has no inverse). Normalizing by requiring the determinant of a diagonal matrix to be the square root of the products of the norms of its elements (which gives the square root of Study's determinant) the Hadamard bound theorem is proved in the form: $\Delta(A) \leqq n^{n / 2} \alpha^{n}$, where the elements of $A$ are real quaternions $a_{i j}$ such that Norm $\left(a_{i j}\right) \leqq \alpha$. The stated bound is shown to be attained by specializing the $a_{i j}$ to be complex numbers. If the bound can also be attained by a matrix every element of which is one of the sixteen numbers $( \pm 1 \pm i \pm j \pm k) / 2$, then the Hadamard bound for matrices of order $4 n$ is also attained by a real matrix. (Received March 7, 1950.)

\section{E. H. Hadlock: $A$ method of construction of a properly primitive form.}

A method is derived and stated, whereby, given the integral invariants $\Omega, \Delta$ and possible sets of progressions involving 2 and the odd prime factors of $\Omega$ and $\Delta$, the integral coefficients $a, b, c, r$, and $S$ are determined in the properly primitive form $f=a x^{2}+b y^{2}+c z^{2}+2 r y z+2 S x z$ with determinant $d=\Omega^{2} \Delta \neq 0$ so that $f$ will have associated with it the given invariants and sets of progressions. (Received March 6, 1950.)

\section{E. H. Hopper: The decision problem in the free modulator} lattice.

Let $F L[4], F M[4]$, and $F D[4]$ be the free, the free modular, and the free distributive lattice, respectively, on four generators. Polynomials equal in $F L[4]$ are necessarily equal in $M F[4]$ and $F D[4]$ while inequality in $F D[4]$ implies inequality in the other two lattices. The unsolved decision problem in $F M[4]$ is thus reduced to a question of the equality in $F M[4]$ of pairs of polynomials having equal canonical 
forms in $F D[4]$ and unequal canonical forms in $F L[4]$. This method is used to obtain canonical forms in $F M[4]$ for polynomials of length not greater than 5. Inclusion relations between these polynomials are also determined. (Received March 6,1950.)

269t. Ralph Hull: An algebraic proof of a theorem on the full linear group.

The theorem is: every rational integral representation of the full linear group of dimension $n$ is either homogeneous or else is expressible as a sum of homogeneous rational integral representations. (Cf. Murnaghan, The theory of group representations, Johns Hopkins Press, 1938, p. 45.) (Received March 20, 1950.)

\section{R. J. Levit: Divided differences and some extremal properties of functions on finite sets.}

Let $A_{n}$ be a finite set consisting of elements $a_{0}, \cdots, a_{n}$ of an ordered field $\Gamma_{\omega}$, and consider functions $f(x)$ on $A_{n}$ with values in $\Gamma_{\omega}$. For any nonzero element $c \in \Gamma_{\omega}$ let $\Phi_{n k}$ be the class of all functions $f(x)$ such that $f\left(a_{0}, \cdots, a_{n}\right)=c$, where $f\left(a_{0}, \cdots, a_{n}\right)$ denotes the $n$th divided difference of $f(x)$ with respect to the indicated arguments. This paper has the dual purpose of deriving certain formal algebraic properties of divided differences and then applying them to the determination of the function of $\Phi_{n k}$ which deviates least from zero on $A_{n}$ and also the functions whose divided differences of any given order $k \leqq n$ deviate least from zero on $A_{n}$. (Received March 8, 1950.)

\section{Nathaniel Macon: Some theorems on the approximation of} irrational numbers by the convergents of their continued fractions.

Let $\xi$ be any positive irrational number. Denote by $A_{n} / B_{n}$ the convergents of its expansion as a regular continued fraction, and set $\left|\xi-A_{n} / B_{n}\right|=1 / \lambda_{n} B_{n}^{2}$. Recently Brauer and Macon (Amer. J. Math. vol. 71 (1949) pp. 349-361 and vol. 72 (1950)) obtained the best possible lower bound for the sum of $k$ consecutive $\lambda_{n}$, for every $k$. In this paper, the best possible lower bounds for the products of two, three, and four consecutive $\lambda_{n}$ are obtained. For $k=3$, this generalizes a well known theorem of Borel (J. Math. Pures Appl. (5) vol. 9 (1903) pp. 329-375). Moreover, Brauer and Macon proved that in any sequence of five consecutive $\lambda_{n}$ either at least two are greater than $5^{1 / 2}$ or one is greater than 3 . They obtained similar results for every sequence of $3 m+2$ consecutive $\lambda_{n}$. These results are now improved for eight and eleven consecutive $\lambda$ 's. (Received March 6, 1950.)

272t. Leo Moser: On sets of integers which contain no three in arithmetical progression.

Let $g(n)$ be the maximum number of integers under $n$, no three of which form an arithmetical progression. F. A. Behrend (Proc. Nat. Acad. Sci. U.S.A. (1946) pp. 331333) showed that $g(n)>n^{1-c /(\log n)^{1 / 2}}$, where $c$ is a fixed constant. In Behrend's method the existence of appropriate sets is proved, but these differ for different $n$. An infinite sequence is here constructed, which has no three elements in arithmetical progression, and which has under $n$ at least $n^{1-c /(\log n)^{1 / 2}}$ integers. Upper bounds for $g(n)$ were given by P. Erdös and P. Turán (Proc. London Math. Soc. (1936) pp. 261-264). Their results are here improved to $g(n)<4 n / 11+5$. (Received March 6, 1950.) 


\section{O. M. Nikodým: Remarks on the cartesian product of Boolean} measure algebras.

Kakutani's theorem, stating the possibility of extension of the finitely additive measure of the cartesian product of denumerably additive $\sigma$-fields of sets provided with denumerably additive measures to a denumerably additive measure on the Borelian extension of this product, can be generalized to abstract Boolean measure algebras. Circumstances relating to this extension are discussed. (Received May 8, 1950.)

274. E. B. Shanks: An elementary proof of the fundamental theorem of algebra.

Let $N(t)$ be the net number of complete revolutions made by a line segment (of variable length) joining the origin to the point representing $f(z)=z^{n}+a_{1} z^{n-1}+\cdots$ $+a_{n}$ (where $n$ is a positive integer and $a_{n} \neq 0$ ) when $z$ traces out the circle $|z|=t$ one time. It is shown (1) if $t=t_{1}$ is sufficiently small, then $N\left(t_{1}\right)=0$; (2) if $t=t_{2}$ is sufficiently large, then $N\left(t_{2}\right)=n$ (this is proved in What is mathematics?, Courant and Robbins, Oxford University Press, New York, 1941, p. 271); (3) if $N(t)$ is not a constant for all $t$ such that $0 \leqq T_{1} \leqq t \leqq T_{2}$, then $f(z)=0$ for some $z$ in the annular region $0 \leqq T_{1} \leqq|z| \leqq T_{2}$. It follows from these three lemmas that $f(z)=0$ for some $z$ in the annular region $0 \leqq t_{1}$ $\leqq|z| \leqq t_{2}$. (Received March 3, 1950.)

\section{J. A. Ward: A function of $k$ constants.}

Let $C_{1}, C_{2}, \cdots, C_{k}$ be an arbitrary set of $k$ constants over a field $F$, with $C_{j}=0$ for $j>k$. For $p \geqq 0$ and $n$ integers the function $W_{p, n}$ is defined as follows: (1) For $n>0, W_{0}, n=\sum C_{i_{1}} C_{i_{2}} \cdots C_{i_{t}}$ where $\sum$ denotes the sum of products obtained by all permutations of all sets of $i$ 's $(i=1,2, \cdots, k)$ such that $i_{1}+i_{2}+\cdots+i_{t}=n$; (2) for $n>0, W_{p, n}$ is defined to be the result obtained from $W_{0, n}$ by increasing by $p$ the first subscript in each term of $W_{0, n}$; (3) if $n \leqq 0, W_{p, n}=\delta_{p},-n$. It then follows for all $i$ and $n$ that $C_{i} W_{0, n}+W_{i, n}=W_{i-1, n+1}$; and other similiar relations are derived. Typical applications of the $W_{p, n}$ are: If $\theta$ satisfies the equation $X^{k}-\sum_{i=1}^{k} C_{i} X^{k-1}=0$, then for all $j \geqq 0, \theta^{i}=\sum_{t=0}^{k-1} W_{t, i-k+1} \theta^{k-1-t}$. Also if $A$ is the companion matrix of the above equation, then for all $i \geqq 0, A^{i}=\left(W_{k-r, i+s-k}\right)$, where $r$ and $s$ are the row and column indices, respectively. (Received March 8, 1950.)

\section{ANALYSIS}

\section{W. L. Duren: Extension of the Daniell-Stone integral to vector-} valued functions.

M. H. Stone in Proc. Nat. Acad. Sci. U.S.A. vol. 34 (1948) has redeveloped the Daniell general integral arising from a given elementary integral. Stone's treatment, in common with other treatments of the Daniell integral, develops the theory for real-valued functions. The purpose of the present paper is to show that the Stone theory may be extended to functions whose values lie in a Banach space. The additional restrictions required for this extension are that, for functions $f$ in the elementary class and their elementary integrals $E(f),\|E(f)\| \leqq \int\|f(x)\| d x$, the latter integral being required to exist as a general integral of a real-valued function. (Received March 9, 1950.) 
277. F. A. Ficken: Severely singular integral operators and Hölder continuity. Preliminary report.

In discussing second order partial differential equations of elliptic type, one may encounter integral operators of the form $u \rightarrow Q u=\int q\left(R, R^{\prime}\right) u\left(R^{\prime}\right) d v_{R^{\prime}}$, where $R$ and $R^{\prime}$ are position vectors of points in $n$-dimensional Euclidean space $E_{n}, d v_{R^{\prime}}$ is the element of volume at $R^{\prime}$, and the integration is extended over $E_{n}$. The kernel $q\left(R, R^{\prime}\right)$ and the operator $Q$ are said to be severely singular if $q\left(R, R^{\prime}\right)=q\left(R-R^{\prime}\right)$, where $q(R)$ is continuous for $R \neq 0$ and behaves like $|R|^{-n}$ as $R \rightarrow 0$ or $R \rightarrow \infty$. It is assumed further that: (i) if $|R| \leqq\left|R^{\prime}\right|$, then, for some positive constant $K,\left|q\left(R^{\prime}\right)-q(R)\right|$ $\leqq K|R|^{-n}\left|R^{\prime}\right|^{-1}\left|R^{\prime}-R\right|$ and (ii) $\int q(R) d v_{R}=0$ if the integral is extended through any spherical shell $0<a \leqq|R| \leqq b$. As a special case, $q\left(R-R^{\prime}\right)$ has these properties if it is the second derivative of $\left|R-R^{\prime}\right|-n+2$. With a view to iterative methods it is useful to know, under these circumstances, that if $u$ is Hölder continuous, then $Q u$ is Hölder continuous. The object of the present note is to give for this fact a proof believed to be simpler than those now available. Simplification results mainly from the observation that $\int\left[g\left(R^{\prime}-S\right)-q(R-S)\right] d v_{S}$, extended over a half-space $|X-R|>\left|X-R^{\prime}\right|$, exists and has an upper bound that is independent of $R$ and $R^{\prime}$. (Received March 16, 1950.)

\section{A. K. Hinds and W. M. Whyburn: Characteristic sets for a} non-self-adjoint second order differential system.

The second order differential system $y^{\prime}=K(x, \lambda) z, z^{\prime}=G(x, \lambda) y, \psi(a, \lambda)=0$, $\phi(a, \lambda)=\phi(b, \lambda), \psi(x, \lambda)=\gamma(x, \lambda) z(x, \lambda)-\delta(x, \lambda) y(x, \lambda), \phi(x, \lambda)=\alpha(x, \lambda) z(x, \lambda)$ $-\beta(x, \lambda) y(x, \lambda)$, is examined for existence and and oscillation theorems. Under mild hypotheses on the coefficients, it is shown that there exist sets $S_{1}, S_{2}, \cdots, S_{n}$ of characteristic lambda values such that the given differential system is compatible for each lambda in these sets. Furthermore, the sets are ordered in the sense that any number in $S_{i}$ is less than any number in $S_{j}$ when $i<j$. Oscillation theorems are established and separation theorems are developed for solution functions associated with two characteristic numbers that belong to the same $S_{i}$. (Received February 24,1950.)

\section{9t. J. D. Mancill: Identically non-regular problems in the calculus of variations.}

This paper is concerned with simple problems of the calculus of variations whose integrand $f\left(x, y, y^{\prime}\right)$ satisfies the identity $f_{y^{\prime} y^{\prime}}\left(x, y, y^{\prime}\right) \equiv 0$ in a closed region $R$ of the $x y$-plane and for all finite values of $y^{\prime}$, from which it follows that $f\left(x, y, y^{\prime}\right) \equiv P(x, y)$ $+y^{\prime} Q(x, y)$. Since Euler's condition for this problem has the form $P_{y}=Q_{x}$, either it is an identity in $R$ and no proper minimum exists if the end points are fixed; or Euler's equation is not an identity and is either never satisfied or is satisfied only along certain paths. In the latter case, given two points of the region $R$ there will not in general exist an extremal joining these points. For this reason one is likely to assume that this problem contains little of interest. That such is not the case follows, in the author's opinion, from the very definite properties of a curve which does furnish a minimum. Also, when the end points are on the boundary of $R$, the necessary conditions are more generally satisfied. Furthermore, this whole problem is an excellent example in contrast to the well known results for regular problems. A minimum cannot exist for the analogous problem in parametric representation unless $P_{y}=Q_{x}$ identically. (Received March 9, 1950.) 
280t. K. S. Miller: A Sturm-Liouville problem associated with iterative methods.

The problem considered in this paper is that of estimating rates of convergence in solving linear ordinary differential equations by an iterative process. Consider a linear differential operator $M$ of the $(n-2)$ th order and associated two point boundary conditions $U_{\alpha}(u), \alpha=1,2, \cdots, n-2$, such that the totally homogeneous system $M u=0$, $U_{\alpha}(u)=0$ is incompatible. Then a sequence of iterants $u_{0}(x), u_{1}(x), u_{2}(x), \cdots$ is constructed such that $u_{m}(x)$ converges to a solution of the differential system as $m$ tends to infinity. Let $M u_{m}(x)=\epsilon_{m}(x)$. Then it is shown that there exists a transformation $Q$, whose inverse is a Sturm-Liouville system, such that $\epsilon_{m}(x)=Q \epsilon_{m-1}(x)$. This problem is solved yielding a complete orthonormal set of functions $\left\{\psi_{n}(x)\right\}$ and associated characteristic number $\left\{\Lambda_{n}\right\}$. It is then shown that $\epsilon_{0}(x)=\sum a_{\alpha} \psi_{\alpha}(x)$ where all the $\psi_{\alpha}(x)$ appearing in the sum have characteristic numbers $\Lambda_{\alpha}$ such that $\left|\Lambda_{\alpha}\right|<1$. Hence $\left\|\epsilon_{m}(x)\right\|^{2}=\sum a_{\alpha}^{2} \Lambda_{\alpha}^{2 m}$ and the rapidity of convergence can be determined explicitly without actually calculating the $u_{\alpha}(x)$. (Received February 27, 1950.)

281. L. V. Robinson: Solution of linear second-order partial differential equations by operator methods.

By making use of the differential operator, $\lambda \equiv p_{1}\left(x_{1}, x_{2}, \cdots, x_{n}\right) \partial / \partial x_{1}+p_{2}\left(x_{1}, x_{2}\right.$, $\left.\cdots, x_{n}\right) \partial / \partial x_{2}+\cdots+p_{n}\left(x_{1}, x_{2}, \cdots, x_{n}\right) \partial / \partial x_{n}$, it is shown how second-order linear partial differential equations can be solved. Conditions necessary for the solution of such equations are also derived. (Received March 9, 1950.)

\section{W. S. Snyder: A characterization of set functions with derivative zero a.e.}

A necessary and sufficient condition is derived for a set function to have derivative 0 a.e. Let $f$ be real-valued and defined on an arbitrary class $\Gamma$ of closed sets lying in a bounded region of the plane. $\mathcal{E}$ denotes generically a finite class of disjoint sets of $\Gamma$, and $\dot{\varepsilon}$ is the point set they cover. $\|\mathcal{E}\|=\max \{$ diameter $T\}$ for $T \in \mathcal{E}$. The derivative in question $D(f, x)=\lim _{n} f\left(\varepsilon_{n}\right) /\left|\mathcal{E}_{n}\right|$ where $f\left(\mathcal{E}_{n}\right)=\sum f(T)$ for $T \in \mathcal{E}_{n}$ and $\left\{\mathcal{E}_{n}\right\}$ is any sequence such that $\left\{\dot{\mathcal{E}}_{n}+x\right\}$ is regular, and diameter $\left\{\dot{\mathcal{E}}_{n}+x\right\} \rightarrow 0$. A necessary and sufficient condition that $D(f, x)=0$ a.e. is that given $\epsilon>0$ there exists a $\delta>0$ such that $\|\mathcal{E}\|<\delta$ implies the existence of a subclass $\mathcal{E}_{1} \subset \mathcal{E}$ for which $\left|\dot{\varepsilon}-\dot{\varepsilon}_{1}\right|<\epsilon$ and $|f|\left(\varepsilon_{1}\right)<\epsilon$. When $\bar{D}(f, x)$ can be defined a.e., a necessary and suffcient condition that $D(f, x)$ exist and be summable is that $f(T)=g(T)+h(T)$, where $g(T)=\int_{T} D(g, x)$ and $D(h, x)=0$ a.e. In particular, if $f$ is B.V. and $D(f, x)$ exists a.e., then $D(f, x)$ is summable and the previous decomposition generalizes the Lebesque decomposition without additivity for $f$. (Received March 6, 1950.)

\section{Applied Mathematics}

283. W. R. Mann: On a class of nonlinear integral equations of the Volterra type. Preliminary report.

Certain nonlinear boundary value problems arising in the study of heat transfer and diffusion phenomena can be expressed in terms of nonlinear integral equations of the form $y(t)=\int_{0}^{t} K(t, \tau) G[y(\tau)] d \tau$ where $G$ is continuous and strictly decreasing in $y$, and $K(t, \tau)$ is positive and increasing in $\tau$ for all $t$. Using Schauder's generalization 
of the Brouwer fixpoint theorem to Banach spaces, it is easily shown that this equation has a solution, $y(t)$, for all $t \geqq 0$. From the proof of this existence theorem, important bounds for $y(t)$ can also be derived. If one assumes that $G$ satisfies a Lipschitz condition and that the $n$th iterated integral of $K(t, \tau)$ with respect to $\tau$ from 0 to $t$ tends to zero sufficiently rapidly for all $t$, as $n \rightarrow \infty$, a set of successive approximations $\left\{y_{n}(t)\right\}$ is defined which have the important property that for all $t>0, y_{1}>y_{3}$ $>y_{5}>\cdots>y_{2 n+1}>\cdots>y(t)$, and $y_{0}<y_{2}<y_{4}<\cdots y_{2 n}<\cdots y(t)$. From these inequalities, a good estimate for the error in the $n$th approximation is easily found. (Received February 24, 1950.)

284. W. R. Mann and J. H. Roberts: Concerning a certain integral equation.

Mann and Wolf considered the equation (1): $y(t)=\int_{0}^{t}\left\{G[y(\tau)] /[\pi(t-\tau)]^{1 / 2}\right\} d t$ and showed that if $G(y)$ is continuous and decreasing for $y \geqq 0$, and $G(1)=0$, then (1) has at least one solution. Under the additional assumption that $G$ satisfies a Lipschitz condition on the interval $[0,1]$, they proved that the solution of (1) was unique and nondecreasing. In the present paper the same conclusion is obtained without the extra hypothesis on $G$. Furthermore the function $[\pi(t-\tau)]^{1 / 2}$ can be replaced by any function $K(t-\tau)$ which, for $x>0$, is continuous, positive, decreasing, and convex, and such that $\lim \int_{\epsilon}^{1} K(x) d x$, as $\epsilon \rightarrow 0_{+}$, is finite. For example, instead of the one-half power in the denominator of the integrand of (1), we can take any power $k$ such that $0<k<1$, and the resulting equation has a unique solution $y(t)$, which is strictly increasing and such that $y(t) \rightarrow 1$ as $t \rightarrow \infty$. (Received March 9,1950.)

\section{E. K. Ritter: $A$ method for computation of normal trajectories of missiles.}

The normal equations of motion of the center of mass of a self-propelled missile referred to a pair of rectangular axes with origin on the earth's surface, can be transformed through the introduction of drag, lift, and thrust functions similar to the conventional drag function of artillery ballistics. The transformed equations may readily be solved by numerical techniques, applicable alike to "hand" computation and to computation by high-speed, automatic calculating machines. (Received April 11, 1950.)

\section{C. B. Smith: Effect of a change in moisture content on the elastic} behavior of plywood plates.

This investigation is concerned with the elastic behavior of a plywood plate subjected to a uniform change in moisture content. By means of a mathematical analysis the distribution of stress is found for infinite plates of two and three plies. This distribution of stress is fairly simple, for all the stress components vanish except the normal stress components parallel to the surfaces of the plate. These are found to be discontinuous at the planes of separation of adjacent plies in both types of plates studied. The distribution of stress is found to be linear in each ply in the twoply plate, and constant in each ply of the three-ply plate. (Received March 3,1950.)

\section{GEOMETRY}

287. J. M. Clarkson: A vector representation of the regular polyhedrons. 
The five regular polyhedrons are related in the following manner: the tetrahedron is such that vectors from the center of the circumscribed sphere to the centroids of the faces meet the sphere, when they are multiplied by a suitable scalar, in the vertices of a second tetrahedron; the same relationship between the cube and the regular octahedron exists, and so does that between the dodecahedron and the icosahedron. This paper describes the vector representation of the vertices of the polyhedrons, and derives the scalar multipliers for the vectors to the centroids of the faces. As a byproduct, the representation, by radicals, of the trigonometric functions of certain angles not multiples of $30^{\circ}$ or $45^{\circ}$ are obtained. (Received May 8,1950.)

\section{Patrick Du Val: Regular surfaces of genus three.}

The main theorem is that the general surface of order $4 n$ in $n+3$ dimensions defineable as the intersection of the intersection of the cone projecting a Veronese surface from an $n-3$ dimensional space, with a fivefold variety whose general surface section is the del Pezzo surface of order $n$, is the bicanonical model of a regular surface of superficial genus 3 and linear genus $n+1$. For $n \leqq 5$, this is the most general surface of its genera; and for any $n$, the bicanonical model of every surface of these genera lies on the Veronese cone, its canonical system being traced by the net of quadric cones on the latter. The canonical model is an $n$-ple plane, with branch curve of order $4 n$, having $24(n-2)$ cusps and $8(n-2)(n-3)$ nodes. For $n \leqq 3$, a complete specification of the branch curve is already known (P. Du Val, J. London Math. Soc. vol. 8 (1933) p. 14). For $n=4$, it is the envelope of a rational family of quartics, of index 3 , of which two reduce to repeated conics; and every such envelope is the branch curve of a canonical surface of these genera. (Received February 25, 1950.)

289. G. B. Huff: Cremona's equations and the properness inequalities. II. Preliminary report.

In part I (Bull. Amer. Math. Soc. Abstract 55-7-314) it was shown that all solutions of Cremona's equations and the properness inequalities are indeed proper for $p=0,1$ and $d>0$. In the present paper, a substantial generalization of Noether's inequality is obtained, and it is shown that, for any particular $p(p \geqq 2)$, the question as to whether the properness inequalities do imply that a solution of Cremona's equations is actually proper can be settled by examining a finite number of cases. In particular, a check is obtained on tables constructed by Du Val (Proc. Lond. Math. Soc. (2) vol. 35, Part 1) and the result of part I is found to be true for $p=2$. (Received March 6, 1950.)

\section{0t. Jack Levine: Collineations in generalized spaces. I.}

The general space of paths is characterized by its equations $d^{2} x^{i} / d t^{2}+H^{i}(x, d x / d t)$ $=0$ defining the paths, where $H^{i}$ are homogeneous of the second degree in $d x^{i} / d t$ (M. S. Knebelman, Amer. J. Math. vol. 51 (1928) pp. 527-564). It is shown that the equations of affine collineations $\Delta \Gamma_{j k}^{i}=0$ obtained by Davies (J. London Math. Soc. vol. 18 (1943) pp. 100-107) can be simplified to $\Delta H^{i}=0$, where $\Delta$ represents the Lie derivative operator, and $2 \Gamma_{j k}^{i}=\partial^{2} H^{i} / \partial p^{i} \partial p^{i}\left(p^{i}=d x^{i} / d t\right)$. The components $\Delta H^{i}$ are those of a contravariant vector. A complete solution of $\Delta H^{i}=0$ is obtained for $n=2$ using Lie's classification of all real continuous groups $G_{r}$ in two variables. Nine types of generalized two-dimensional space are found admitting groups of affine collineations. The maximum number of parameters is three, there being six spaces admitting $G_{3}$ 's, two admitting $G_{2}$ 's and one admitting a $G_{1}$. These exclude spaces reducing to 
ordinary affine spaces $A_{2}$. These results are an extension of some previous results of a former paper (Bull. Amer. Math. Soc. Abstract 55-7-381t). (Received March 6, 1950.)

\section{TOPOLOGY}

\section{R. H. Bing: Hereditarily indecomposable continua.}

Examples are described showing that the plane contains as many topologically different hereditarily indecomposable compact continua as it does continua. The property of being a chained compact continuum is invariant under a monotone continuous transformation. Using this result and the fact that two nondegenerate hereditarily indecomposable compact continua are homeomorphic if each can be chained, we show that if $N$ is a nondegenerate image of a hereditarily indecomposable compact continuum $M$ under a monotone continuous transformation, then $N$ is homeomorphic to $M$. (Received March 8, 1950.)

\section{W. J. Conner: On locally closed sets.}

A set $A$ of a topological space $X$ is defined to be locally closed at a point $p$ if $p$ has a neighborhood whose closure rela tive to $A$ is closed in $X$ (stronger than the definition given by Kuratowski). "Local closure" and "locally closed set" are defined and the relationship between closure and local closure is used to characterize regular spaces, normal spaces, locally compact subsets of locally compact spaces. (Received March $6,1950$.

\section{L. J. Derr: $A$ theorem on exact sequences.}

Let $G_{1}, G_{2}$ be subgroups of an abelian group $G$. Using the Alexander-Kolmogoroff cohomology groups there exists an exact sequence: $\rightarrow H^{p}\left(X ; G_{1}\right) \times H^{p}\left(X ; G_{2}\right)$ $\rightarrow H^{p}\left(X ; G_{1}+G_{2}\right) \rightarrow H^{p+1}\left(X ; G_{1} \cap G_{2}\right) \rightarrow$ where the groups in the parentheses indicate the coefficient group used in each case. It is clear that $G_{1} \cap G_{2}=0$ implies $H^{p}\left(X ; G_{1}\right)$ $\times H^{p}\left(X ; G_{2}\right) \cong H^{p}\left(X ; G_{1}+G_{2}\right)$. (Received March 8, 1950.)

\section{4t. W. H. Gottschalk: Choice functions and Tychonoff's theorem.}

The purpose of this note is to point out that the following set-theoretic theorem [R. Rado, Axiomatic treatment of rank in infinite sets, Canadian Journal of Mathematics vol. 1 (1949) pp. 337-343] is an easy consequence of Tychonoff's theorem that the cartesian product of a family of compact spaces is compact. Theorem. Let $\left(X_{\alpha} \mid \alpha \in I\right)$ be a family of finite sets, let $\mathfrak{A}$ be the class of all finite subsets of $I$, and for each $A \in \mathbb{Y}$ let $\phi_{A}$ be a choice function of $\left(X_{\alpha} \mid \alpha \in A\right)$. Then there exists a choice function $\phi$ of $\left(X_{\alpha} \mid \alpha \in I\right)$ such that $A \in \mathfrak{A}$ implies the existence of $B \in \mathfrak{A}$ such that $B \supset A$ and $\alpha \phi=\alpha \phi_{B}(\alpha \in A)$. Proof. For $A \in \mathfrak{A}$ let $E_{A}$ be the set of all $\phi \in X=X_{\alpha} \in{ }_{1} X_{\alpha}$ such that $\alpha \phi=\alpha \phi_{B}(\alpha \in A)$ for some $B \in \mathfrak{A}$ with $B \supset A$. Provide each $X_{\alpha}(\alpha \in I)$ with its discrete topology. Since $X$ is compact and $\left[E_{A} \mid A \in \mathfrak{A}\right]$ is a class of nonvacuous closed

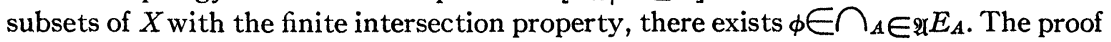
is completed. Corollary. A family of finite sets has a one-to-one choice function if and only if each of its finite subfamilies has a one-to-one choice function. [Cf. C. J. Everett and G. Whaples, Representations of sequences of sets, Amer. J. Math. vol. 71 (1949) pp. 287-293.] (Received January 16, 1950.)

\section{S. T. Hu: Chain transformations in Mayer chain complexes.}

A general theory of the extension and the classification of the equivariant chain 
transformations in Mayer chain complexes with a group $W$ of operators is established in this paper by means of the obstruction methods analogous to those used in the theory of continuous maps. The presentable and the regular subgroups of the equivariant cohomology groups are defined in a natural way, and hence their quotient group $J_{e}^{n}\left(M, H_{n}(N)\right)$, where $M$ and $N$ are Mayer chain complexes with operators $W$ and $H_{n}(N)$ denotes the integral homology group of $N$. The main theorem of classification can be stated as follows: If there are two integers $a$ and $b$ such that $C_{q}(M)=0$ unless $a<q \leqq b$ and if $W$ operates freely on $M$, then the equivariant homotopy classes of the equivariant chain transformations of $M$ into $N$ are in a (1-1) correspondence with the elements of the direct sum $J_{a}^{a+1}\left(M, H_{a+1}(N)\right) \oplus \cdots \oplus J_{e}^{b}\left(M, H_{b}(N)\right)$. A number of specializations are made in the later part of the paper. In particular, for any augmentable closure-finite abstract complex $K$, the condition $H^{q}\left(K, H_{q}(K)\right)=0$ $(0 \leqq q<n)$ implies $H_{q}(K)=0(0 \leqq q<n)$, where $H_{0}(K)$ denotes the reduced 0 th integral homology group. (Received March 6, 1950.)

\section{J. W. Keesee: On a retract of a compact Hausdorff space.}

Let $C(X)$ denote the ring of real continuous functions on the compact Hausdorff space $X$. An ideal $I$ contained in $C(X)$ is called a closed ideal if it is equal to the intersection of all maximal ideals in which it is contained. A compact Hausdorff space $Y$ is homeomorphic to a retract of $X$ if and only if $C(X)$ contains a closed ideal $I$ and a subring $R$ such that $C(X)=I+R, I \cap R=0$, and $R$ is ring-isomorphic to $C(X)$. (Received March 6, 1950.)

\section{V. L. Klee: On certain intersection properties of convex sets.}

A collection of $n+1$ convex subsets of a Euclidean space $E$ will be called an $n$-set in $E$ provided each $n$ of the sets have a common interior point although the intersection of all $n+1$ interiors is empty. It is well known that if $\left\{C_{0}, C_{1}\right\}$ is a 1 -set, then $C_{0}$ and $C_{1}$ can be separated by a hyperplane. In the present note this result is generalized by showing that if $\left\{C_{0}, \cdots, C_{n}\right\}$ is an $n$-set in $E$, then there is a variety $V$ of deficiency $n$ in $E$ such that $V$ intersects no set Int $C_{i}$, although in each direction away from $V, V$ has a translate which intersects some set Int $C_{i}$. This theorem is then used to prove the converse of Horn's recent generalization (Bull. Amer. Math. Soc. vol. 55 (1949) pp. 923-929, Theorem 4) of Helly's theorem on the intersection of convex sets. (Received March 6, 1950.)

\section{A. D. Wallace: Extension and reduction theorems.}

Let $A, B$ be closed subsets of the fully normal (=paracompact) space $X$. Extension Theorem: If $e$ is any element of $H^{p}(B, A \cap B)$ then there is an open set $Q$ containing $B$ and an element $e_{0}$ of $H^{p}(\bar{Q}, A \cap \bar{Q})$ whose image, under the natural homomorphism, is $e$. Reduction Theorem: If the element $e$ of $H^{p}(X, A)$ is in the kernel of the natural homomorphism into $H^{p}(B, A \cap B)$, then, for some open set $Q$ containing $B, e$ is in the kernel of the natural homomorphism into $H^{p}(\bar{Q}, A \cap \bar{Q})$. These are proved for the Alexander-Kolmogoroff cohomology groups. Their validity for normal spaces is an open question. (Received January 30,1950.)

\section{9t. A. D. Wallace: Extensional invariance.}

Theorem: Any completely regular Hausdorff space has a homeomorph $X$ densely contained in a compact Hausdorff space $X^{\prime}$ so that (denoting closure in $X^{\prime}$ by ') if 
$A, B$ are closed in $X$ then $(A \cap B)^{\prime}=A^{\prime} \cap B^{\prime}$ provided that either (i) $X$ is normal or (ii) $X$ is locally compact and one of $A, B$ is compact. This is merely the Tychonoff imbedding (as improved by Cech and Stone) together with the additional result about closure. Case (i) can be deduced from some results of Wallman but case (ii) cannot since Wallman's compaction is not Hausdorff unless $X$ is normal. This result is applied to prove the extensional invariance of certain properties of $X$, for example unicoherence, if $X$ has property S. (Received March 7, 1950.)

W. M. WHYBURN, Associate Secretary 